“C 2013 IEEE. Personal use of this material is permitted. Permission from IEEE must be obtained for all other uses, in any current or future media, including reprinting/republishing this material for advertising or promotional purposes, creating new collective works, for resale or redistribution to servers or lists, or reuse of any copyrighted component of this work in other works." 


\title{
Locational Optimization based Sensor Placement for Monitoring Gaussian Processes Modeled Spatial Phenomena
}

\author{
Linh Van Nguyen, Sarath Kodagoda, Ravindra Ranasinghe, and Gamini Dissanayake \\ Center for Autonomous Systems \\ Faculty of Engineering and IT \\ University of Technology, Sydney, Australia \\ Email: van.1.nguyen-1@ student.uts.edu.au; sarath.kodagoda, ravindra.ranasighe, gamini.dissanayake@uts.edu.au
}

\begin{abstract}
This paper addresses the sensor placement problem associated with monitoring spatial phenomena, where mobile sensors are located on the optimal sampling paths yielding a lower prediction error. It is proposed that the spatial phenomenon to be monitored is modeled using a Gaussian Process and a variance based density function is employed to develop an expected-value function. A locational optimization based effective algorithm is employed to solve the resulting minimization of the expectedvalue function. We designed a mutual information based strategy to select the most informative subset of measurements effectively with low computational time. Our experimental results on realworld datasets have verified the superiority of the proposed approach.
\end{abstract}

\section{INTRODUCTION}

Recent advances in micro-electro-mechanical systems and wireless communications empower wireless sensor networks (WSN) to play a key role in a wide range of modern smart technology applications [1]. In particular, mobile robotic wireless sensor networks have attracted a significant attention for monitoring the spatial phenomena due to its distributed spatial properties and the mobility aspects. For example, applications of this technology includes detecting and monitoring forest fires [2], monitoring air quality [3], and monitoring ocean ecology conditions [4].

With on-board sensors, wireless communication and the mobile ability, the mobile wireless robotic sensor networks are capable of providing services required not only for monitoring and exploring the environment but also for exchanging the information. In [5], Cressie proposed a Gaussian Process (GP) model, also known as Kriging interpolation technique, a nonparametric generalization of linear regression, for the spatial phenomena assuming it obeys a Gaussian distribution. It is possible to exhaustively learn a GP model from the sensor readings and then utilizes this model to predict the values at unobserved locations. Combining these we address the sensor placement problem using the mobile wireless robotic sensor networks. Here, sensing observations and locations of sensors are used to generate a GP model for the phenomena.

Recently, there has been considerable activity in studying the wireless sensor placement problem [6] - [11] and designing wireless sensor networks for optimal monitoring. Nevertheless, most of these works are primarily focused on selecting the best subset from among a set of candidate sensor locations which are selected from the discrete space. For example, in [10], the authors proposed a greedy heuristic algorithm based on entropy based information-theoretic model. The premise behind the entropy approach is to minimize the uncertainty of conditional entropy of unobserved locations, given observations. However, owning to a static sensor network, as shown in [7], the entropy method tends to pick locations along the border of the interested space causing sensed information waste. To address the drawbacks of the entropy approach, Guestrin et al., [7] proposed a new method based on the mutual information. In this method a subset from a set of potential sensor locations is selected such that the mutual information between the selected subset and the rest of the space is maximal. In our previous work [11], a computationally efficient simulated annealing based approximately heuristic algorithm was proposed to solve the sensor selection problem. Nevertheless, solutions of the sensor placement problem, using both mutual information and our previous proposed approach, are not optimal as the subset of sensor locations chosen is fixed.

Motivating on the sensor coverage problem, the authors in [12] proposed an algorithm that allows sensors to concentrate around locations of high event density while satisfying the minimal area coverage constraints. In Cortes et al [13], the authors optimized the mobile sensor network locations with respect to a known event probability density. Their proposed technique guarantees to minimize a cost function locally.

In this paper, we propose to utilize a GP model to develop a variance based density function. The proposed locational optimization based algorithm employed in this work locates the optimal sampling paths for mobile sensors. These trajectories allow to significantly improve the quality of prediction and to reduce the model uncertainty simultaneously. Moreover, a strategy based on mutual information is designed to select the best subset of measurements from all observations. This selection substantially decreases the computation time in developing the density function. In certain circumstances characteristics of the density function may lead the sensors to trap in certain locations. In this case all the mobile sensors are trapped in stationary locations without any further improvement to the sensing quality. An effective technique is developed to drive the mobile sensors to circumcenters instead of moving them to the centroids of the sensor locations' voronoi partitions.

Organization: Section II presents locational optimization 
problem. Section III reviews the basics of Gaussian Process regression. Section IV describes the sensor placement based on the locational optimization. A density function and an algorithm for sensor movements are also analyzed and synthesized here. Experimental results are provided in Section V. Section VI shows the conclusions.

Notation: Let $\mathbb{R}_{+}$be a set of non-negative real numbers. The Euclidean distance function is defined by $\|$.$\| . We let P$ be a convex polytope in $\mathbb{R}^{d}$ and $\operatorname{Ve}(P)$ be a vertex set of $P$. The set of all robotic sensor locations is denoted $S=\left\{s_{1}, \ldots, s_{n}\right\}$. In other words, the position of $i^{t h}$ sensor is denoted $s_{i}$. And we define $p$ as an arbitrary position in $P$. For a set $P$, we let $\mathbb{P}(P)$ denote the collection of subsets of $P$. Other notations will be explained in due course.

\section{LOCATIONAL OPTIMIZATION PROBLEM}

In this section, we describe the basic concepts and results on locational optimization that are used in this work. Readers are referred to [13] and [14] for further details.

\section{A. Voronoi Partition}

Consider a network of $n$ mobile robotic wireless sensors that are deployed in a known, convex polytope $P$. At the fixed locations, the sensors could be divided by the optimal partitions of $P$, which are a set of Voronoi partitions $\mathcal{V}(S)=\left\{V_{1}, \ldots\right.$, $\left.V_{n}\right\} \subset \mathbb{P}(P)$ defined by, for each $i \in\{1, \ldots, \mathrm{n}\}$

$$
V_{i}=\left\{p \in P \mid\left\|p-s_{i}\right\| \leq\left\|p-s_{j}\right\|, \forall j \neq i\right\}
$$

In equivalent words, $V_{i}$ is the set of the positions of $P$ that are closer to $s_{i}$ than to any of the other points in $S$.

\section{B. Centroidal Voronoi Tessellation}

Take a density function (also called bounded measurable function) $\phi: \mathbb{R}^{d} \rightarrow \mathbb{R}_{+}$into a consideration in a bounded environment of interest $P \in \mathbb{R}^{d}$. For this work, we only use the value of $\phi$ limited to $P$ of interest. We can consider $\phi$ as a function of measuring the information of probability that the events happen over $P$. The greater the value of $\phi(p)$ is, the more significant the point $p$ is. In this paper, we use a nonincreasing and piece-wise continuously differentiable function $f_{s}: \mathbb{R}_{+} \rightarrow \mathbb{R}_{+}$as a sensing performance function. This function characterizes the utility at position $p$ taken from $i^{t h}$ mobile robotic wireless sensor at the location $s_{i}$. One can easily see that the value of this function downgrades with the distance $\left\|p-s_{i}\right\|$ between $p$ and $s_{i}$. Therefore, we let this function is defined by a quadratic function $f_{s}=\left\|p-s_{i}\right\|^{2}$.

Given a density function $\phi$, and a sensing performance function $f_{s}$, we formulate the expected-value function over the region $P$ as

$$
\mathcal{H}(S)=\sum_{i=1}^{n} \int_{V_{i}} f_{s} \phi(p) d p
$$

or

$$
\mathcal{H}(S)=\sum_{i=1}^{n} \int_{V_{i}}\left\|p-s_{i}\right\|^{2} \phi(p) d p
$$

From the notation of the Voronoi partition, we can easily be seen that

$$
\begin{aligned}
& \text { minimize }\left\|p-s_{i}\right\|=\left\|p-s_{j}\right\| \text { for all } p \in V_{j}, \\
& \text { subject to } i \in\{1, \ldots, n\} .
\end{aligned}
$$

Therefore, our task here is to minimize the locational optimization function (2)

$$
\operatorname{minimize} \sum_{i=1}^{n} \int_{V_{i}}\left\|p-s_{i}\right\|^{2} \phi(p) d p .
$$

In other words, this optimization problem is to identify an optimal robotic sensor network configuration that makes (2) minimum.

Carrying out the partial derivative of $\mathcal{H}(S)$ with respect to the $i^{\text {th }}$ robotic sensor, we get

$$
\frac{\partial \mathcal{H}(S)}{\partial s_{i}}=\sum_{i=1}^{n} \int_{V_{i}} \frac{\partial}{\partial s_{i}}\left[\left\|p-s_{i}\right\|^{2} \phi(p)\right] d p .
$$

Let us recall in Euclidean space. The generalized area and the first mass moment of a set $P \subset \mathbb{R}^{d}$ with respect to $\phi$ are specified by

$$
A(P)=\int_{P} \phi(p) d p
$$

and

$$
F M(P)=\int_{P} p \phi(p) d p .
$$

Therefore, the centroid (center of mass) of a set $P$ is formulated by

$$
C(P)=\frac{F M(P)}{A(P)} .
$$

Moreover, the polar moment of inertia of $P$ about $s \in P$ is defined by

$$
J(P, s)=\int_{P}\|p-s\|^{2} \phi(p) d p .
$$

From [15], by using Parallel Axis Theorem, we can easily state that

$$
J(P, s)=J(P, C(P))+A(P)\|s-C(P)\|^{2} .
$$

From (2) and (9), using the Parallel Axis Theorem again, we simplify (2) to

$$
\mathcal{H}(S)=\sum_{i=1}^{n} J\left(V_{i}, C\left(V_{i}\right)\right)+\sum_{i=1}^{n} A\left(V_{i}\right)\left\|s_{i}-C\left(V_{i}\right)\right\|^{2},
$$

and (5) to

$$
\frac{\partial \mathcal{H}(S)}{\partial s_{i}}=2 A\left(V_{i}\right)\left(s_{i}-C\left(V_{i}\right)\right)
$$

Equation (12) demonstrates that the solution of the locational optimization problem (4) is the set of centroidal Voronoi configurations in $P$ [16]. In other words, the optimal mobile robotic wireless sensor location is the centroid of its Voronoi cell. 


\section{Gaussian Process Background}

Consider a two dimensional robotic sensor network that has a $\mathcal{Q},|\mathcal{Q}|=m$, set of possible locations, which provide point measurements of some physical quantities. If we deploy all mobile sensors in $\mathcal{Q}, S \subset \mathcal{Q}$, sensor readings can still represent the distribution of the physical quantity in the whole space. This can be effectively predicted using Gaussian Process (GP) [17]. Lets denote $\mathcal{Q}=\left[q_{1}, q_{2}, \ldots, q_{m}\right]$ as a set of locations, and $\mathcal{Z}_{\mathcal{Q}}=\left[z_{1}, z_{2}, \ldots, z_{m}\right]$ as corresponding random variables at these locations. A joint probability distribution is given by

$$
P\left(Z_{\mathcal{Q}}=\mathbf{z}_{\mathcal{Q}}\right)=\frac{1}{(2 \pi)^{n / 2} \Sigma_{\mathcal{Q} \mathcal{Q}}} e^{-\frac{1}{2}\left(\mathbf{z}_{\mathcal{Q}}-\mathbf{m}_{\mathcal{Q}}\right)^{T} \Sigma_{\mathcal{Q} \mathcal{Q}}^{-1}\left(\mathbf{z}_{\mathcal{Q}}-\mathbf{m}_{\mathcal{Q}}\right)},
$$

where $\mathbf{m}_{\mathcal{Q}}$ is the mean vector and $\Sigma_{\mathcal{Q Q}}$ is the covariance matrix of random variables, $\mathcal{Z}_{\mathcal{Q}}$.

Rasmussen et al. [17] demonstrated that GP has a marginalization property, which implies for any subset, $S$, of $\mathcal{Q}$, the joint distribution on random variables of its locations is Gaussian. Moreover, GP is comprehensively specified by its mean function $\mathcal{M}(q)=\mathbb{E}[z(q)]$, and a symmetric positive definite covariance function $\mathcal{C}\left(q_{1}, q_{2}\right)$, often called kernel function. One of frequently used kernel function is squared exponential, i.e.,

$$
\mathcal{C}\left(q_{1}, q_{2}\right)=\sigma_{f}^{2} e^{-\frac{\left\|q_{1}-q_{2}\right\|^{2}}{2 l^{2}}},
$$

where $q_{1}, q_{2} \in \mathcal{Q}, \sigma_{f}^{2}$ is the maximum allowable covariance, $l$ is the bandwidth of the kernel, and $\left\|q_{1}-q_{2}\right\|^{2}$ denotes the Euclidean distance between $q_{1}$ and $q_{2}$.

We define $S \subset \mathcal{Q}$ as a subset that includes all the selected sensors locations. For any subset $S$, let $\mathcal{Z}_{S}$ denote the collections of observations at locations in $S$. In addition, take into account $\mathcal{U}=\mathcal{Q} \backslash S$ as the set of all elements in $\mathcal{Q}$ but not in $S$, and $\mathcal{Z}_{\mathcal{U}}$ is a vector of random variables over these unobserved locations. We will assume that sensor measurement has an additive independent identically distributed zero-mean Gaussian noise with variance $\sigma_{n}^{2}$. It can be clearly shown that $\mathcal{Z}_{S}$ and $\mathcal{Z}_{\mathcal{U}}$ are jointly Gaussian distributed as

$$
\left[\begin{array}{l}
\mathcal{Z}_{S} \\
\mathcal{Z}_{\mathcal{U}}
\end{array}\right] \sim \mathcal{N}\left(\left[\begin{array}{l}
\mathbf{m}_{S} \\
\mathbf{m}_{\mathcal{U}}
\end{array}\right],\left[\begin{array}{cc}
\Sigma_{S S}+\sigma_{n}^{2} I & \Sigma_{S \mathcal{U}} \\
\Sigma_{\mathcal{U} S} & \Sigma_{\mathcal{U U}}
\end{array}\right]\right)
$$

where $\mathbf{m}_{S}$ and $\mathbf{m}_{\mathcal{U}}\left(\Sigma_{S S}\right.$ and $\left.\Sigma_{\mathcal{U U}}\right)$ are mean vectors (covariance matrices) of $\mathcal{Z}_{S}$ and $\mathcal{Z}_{\mathcal{U}}$, respectively. $\Sigma_{\mathcal{U} S}\left(=\Sigma_{S \mathcal{U}}^{T}\right)$, are cross-covariance matrices between $\mathcal{Z}_{S}$ and $\mathcal{Z}_{\mathcal{U}}$; and $I$ is the $|S| \times|S|$ identity matrix.

In probabilistic terms, we derive the conditional distribution at predicted positions of $\mathcal{U}$, given $\mathcal{Z}_{S}$ as follows:

$$
\begin{gathered}
\mathbf{m}_{\mathcal{U} \mid S}=\mathbf{m}_{\mathcal{U}}+\Sigma_{\mathcal{U} S}\left(\Sigma_{S S}+\sigma_{n}^{2} I\right)^{-1}\left(\mathcal{Z}_{S}-\mathbf{m}_{S}\right), \\
\Sigma_{\mathcal{U} \mid S}=\Sigma_{\mathcal{U} \mathcal{U}}-\Sigma_{\mathcal{U} S}\left(\Sigma_{S S}+\sigma_{n}^{2} I\right)^{-1} \Sigma_{S \mathcal{U}},
\end{gathered}
$$

where $\mathbf{m}_{\mathcal{U} \mid S}$ and $\Sigma_{\mathcal{U} \mid S}$ are mean vector and covariance matrix of $\mathcal{Z}_{\mathcal{U}}$, given $\mathcal{Z}_{S}$. As a consequence, using observations at locations in set $S$, we can predict quantities at unobserved locations, $\mathcal{U}$. This process is described as the Gaussian regression approach [18].

The quality of prediction is generally measured by calculating the errors at unobserved locations. Therefore, the goal is to drive robotic sensors to optimal locations $S \subset \mathcal{Q}$ so that it will minimize a certain measure of prediction error at unobserved locations, given observations $\mathcal{Z}_{S}$. A typically used function inspired by the notions of G-optimality from optimal design [5], [19] is maximum predictive variance. Specifically, it is given by

$$
\begin{aligned}
\mathcal{M}(\mathcal{U})= & \text { maximize } \sigma_{f}^{2}-\Sigma_{q S}\left(\Sigma_{S S}+\sigma_{n}^{2} I\right)^{-1} \Sigma_{S q} \\
& \text { subject to } q \in \mathcal{U}
\end{aligned}
$$

where $\Sigma_{q S}\left(=\Sigma_{S p}^{T}\right)$ is the cross-covariance column vector between $\mathcal{Z}_{S}$ and $z_{q}$.

\section{LOCATIONAL Optimization Based Sensor Placement}

\section{A. Density Function}

Recall that in section II, we employed the density function to describe the measurement of information or probability of the events over $P$. In this work, we developed a density function based on the variances of the random variables $\mathcal{Z}_{\mathcal{Q}}$.

Consider a location $p \in \mathcal{U}, \mathcal{U} \subset \mathbb{R}^{2}$. From (17), the predicted variance at location $p$, given observations generated by $S$, is specified by

$$
\Sigma_{p \mid S}=\Sigma_{p p}-\Sigma_{p S}\left(\Sigma_{S S}+\sigma_{n}^{2} I\right)^{-1} \Sigma_{S p},
$$

where $\Sigma_{p p}=\sigma_{f}^{2}$, and $\Sigma_{p S}\left(=\Sigma_{S p}^{T}\right)$ is the cross-covariance column vector between $\mathcal{Z}_{S}$ and $z_{p}$.

In this paper, we embedded $P$ into a two dimensional space, and we define

$$
\begin{gathered}
\phi(p)=\Sigma_{p \mid S} \\
\phi(p)=\Sigma_{p p}-\Sigma_{p S}\left(\Sigma_{S S}+\sigma_{n}^{2} I\right)^{-1} \Sigma_{S p} .
\end{gathered}
$$

The actual measurements substantially influence on the density function as the covariance matrix include the hyperparameters that are learned from the actual sensor readings.,. Therefore when mobile robotic wireless sensors get to new positions, they need to sample the environment to generate a new density function.

\section{B. Computations}

There are two key issues associated with the implementation of the proposed algorithm in real mobile robotic wireless sensor networks: (a) limited computing resources to continuously compute the density function (b) moving mobile sensors out of stationary locations due to undesirable characteristics of the density function. As it requires to compute the density function in real-time after each iteration and it depends on the number of observations, calculation of this density function becomes intractable with increasing number of sensors' readings. 
1) Mutual Information: In order to address the first issue, we employed a method based on the mutual information, a concept from information theory [20], to select the most informative subset of measurements from among all of the potential sensor measurements. After that the selected subset is utilized to develop the density function. For instance, to find $k$ best measurements from whole observations $\mathcal{O}$, it starts from an empty set of locations $\mathcal{A}=\oslash$ and greedily adds placements in sequence until $|\mathcal{A}|=k$. The algorithm chooses the next point that produces the maximum increase of mutual information. More specifically, the mutual information between the subset $\mathcal{A}$ and the rest of observations $\mathcal{O} \backslash \mathcal{A}$ can be formulated by

$$
F(\mathcal{A})=I(\mathcal{A} ; \mathcal{O} \backslash \mathcal{A})
$$

Once $y \in \mathcal{O A}$ is chosen and added to $\mathcal{A}$, the variation of mutual information can be calculated by

$$
\begin{aligned}
& F(\mathcal{A} \cup y)-F(\mathcal{A})= \\
& =H(\mathcal{A} \cup y)-H(\mathcal{A} \cup y \mid \overline{\mathcal{A}})-[H(\mathcal{A})-H(\mathcal{A} \mid \overline{\mathcal{A}} \cup y)] \\
& =H(y \mid \mathcal{A})-H(y \mid \overline{\mathcal{A}})
\end{aligned}
$$

where $\mathcal{A} \cup y$ denotes the $\mathcal{A} \cup y$ and $\overline{\mathcal{A}}$ represents $\mathcal{O} \backslash(\mathcal{A} \cup y)$; $H(y \mid \mathcal{A})$ is the conditional entropy of y after observing $\mathcal{A}$. By maximizing $F(\mathcal{A} \cup y)-F(\mathcal{A})$, we will find the next point $y$ to add into $\mathcal{A}$. Suppose that the variables in $\mathcal{O}$ are distributed normally, then 22 can be calculated in detail by

$$
\begin{aligned}
& F(\mathcal{A} \cup y)-F(\mathcal{A})= \\
& =\frac{1}{2}\left[\log \left(\sigma_{y \mid \mathcal{A}}^{2}\right)-\log \left(\sigma_{y \mid \overline{\mathcal{A}}}^{2}\right)\right],
\end{aligned}
$$

where $\sigma_{y \mid \mathcal{A}}^{2}\left(\sigma_{y \mid \overline{\mathcal{A}}}^{2}\right)$ is the variance of $y$, given $\mathcal{A}(\overline{\mathcal{A}})$.

2) Circumcenter: As discussed in section II-B, the centroid of a sensor's current location will be the sensor's new location in the next movement. Importantly, computation of the centroids substantially depends on the shape of the density function. For instance, if the shape of the current density function is quite similar to that of the previous one or the shape of the density function is nearly flat, the distance between current and new locations is trivial. This leads the mobile robotic wireless sensors to stationary positions, which inadvertently indicates that the mobile sensors have already converge to their optimal locations. In order to address this issue, we utilized an approach derived from a circumcenter notion (refer [21] for more detail). In the event of all mobile robotic wireless sensors are immobile during any iteration without reaching the criterion of sensing quality, mobile sensors are driven from current positions to circumcenters rather than to the centroids of their voronoi cells.

The circumcenter of a bounded set $D \subset \mathbb{R}^{2}$ is the center of the circle of minimum radius that contains $D$. Hence, the circumcenter is unique. The calculation of the circumcenter of a polygon $D \subset \mathbb{R}^{2}$ is to minimize the radius $r$ of the circle centered at $p$ subject to the constraints that the distance between $p$ and each of the polygon vertices is smaller than or equal to $r$. Mathematically, the problem can be formulated as

minimize $r$

subject to $\|p-d\|^{2} \leq r^{2}$, for all $d \in \operatorname{Ve}(D)$

\section{Sensor Movement}

Let us define the dynamics of each sensor movement by employing the first order form:

$$
\dot{s}_{i}=u_{i}
$$

Solving the locational optimization in section II with the density function built in section IV-A, we can find the centroidal Voronoi configuration for a robotic sensor network. As a result, we propose a control law for each mobile sensor as follows:

$$
u_{i}=a\left(s_{i}-C\left(V_{i}\right)\right),
$$

where $a$ is a proportional control gain.

Applying this control input, sensors will move to the new locations that generate constantly the Voronoi partitions.

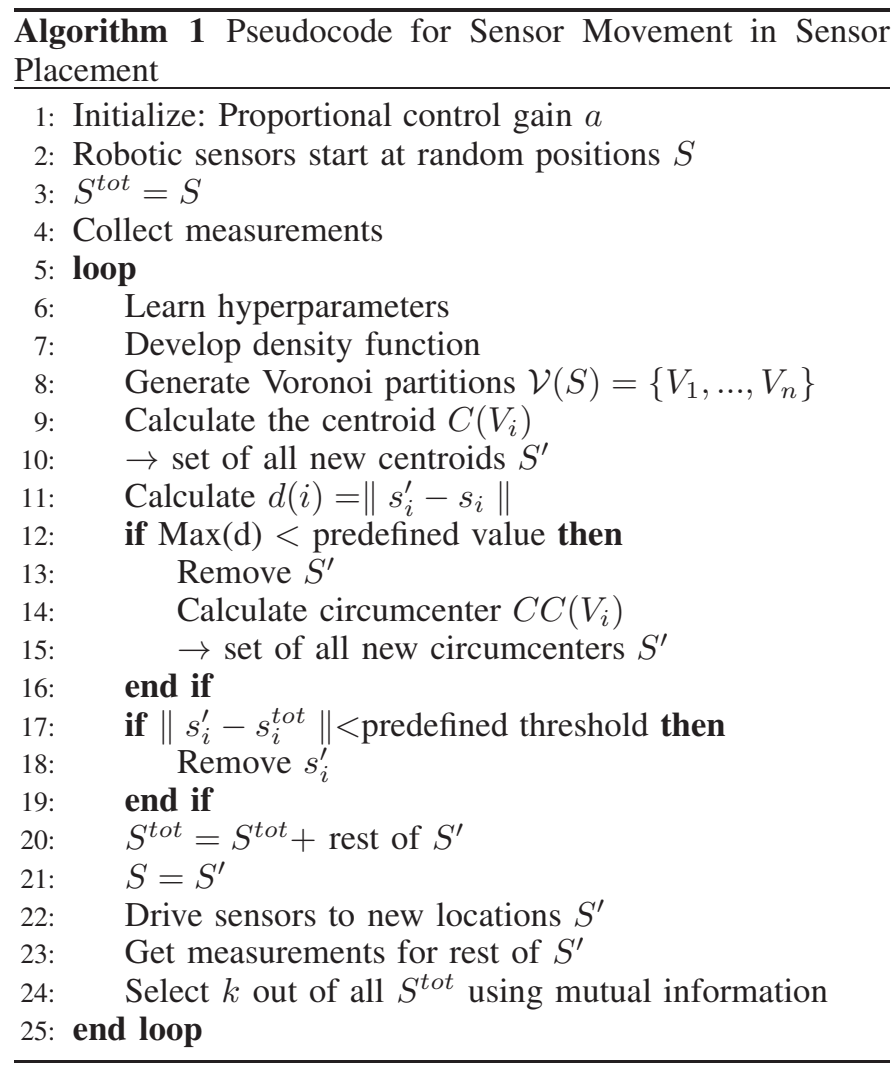

The approach to implement the sensor movements in practice in described by algorithm 1 .

In this paper, we consider the environments that are constant or slowly changing in a short period of time. In this interval, mobile sensors will collect data, compute the new locations based on the locational optimization and move to these new positions, which have high variances. These movements make the maximum predictive variance (18) to gradually decline. Iteratively, sensors will run on the optimal trajectories that result in minimizing the maximum predictive variance.

\section{EXPERIMENTS AND RESULTS}

We conducted experiments using a real world data set of a indoor temperature environment from 


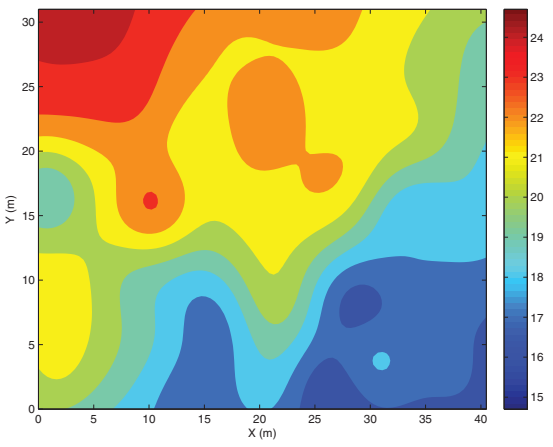

Fig. 1: Spatial temperature field

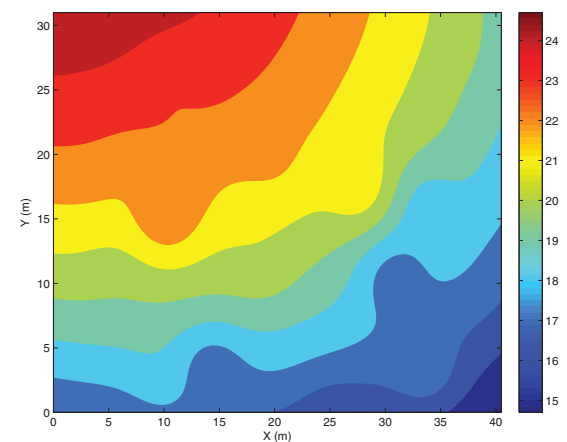

Fig. 2: Predicted temperature field at iteration 1

the Intel Berkeley Research Lab [7] (available at http://db.scal.mit.edu/labdata/labdata.html. Fig. 1 illustrates the spatial temperature field generated using this dataset with 52 sensors.

In this paper, in order to learn a Gaussian Process model, we first choose the isotropic square exponential covariance function and the second order trend for the mean function. One can recall that the covariance function is specified by the set of hyper-parameters $\left(\sigma_{f}, l, \sigma_{n}\right)$. Theoretically, these hyperparameters could be learned by maximizing the log of the marginal likelihood (see [17] for more details). However, in this experiment, we used the geoR package [22] to learn these parameters.

In this experiment, we employ 20 mobile robotic wireless sensors to collect measurements iteratively, starting from random locations as shown in Fig. 5 (in white dots). In this implementation the time period of each iteration is dependent on the configuration of the sensor network, the computational time and the motion time. The predicted temperature fields are demonstrated from Fig. 2 to Fig. 4 at iterations 1, 5, 10. It can be clearly seen that the predictive means are getting closer to the true temperature field Fig. 1 as the number of observations increases. White circles in Figures Fig. 5 to Fig. 7 show the trajectories for the optimal paths of the mobile robotic wireless sensors at iterations 1, 5, and 10. Additionally, Figures Fig. 5 to Fig. 7 also show the prediction error variance corresponding to $1^{\text {st }}, 5^{\text {th }}$ and $10^{\text {th }}$ iterations. Note that the white dots in these

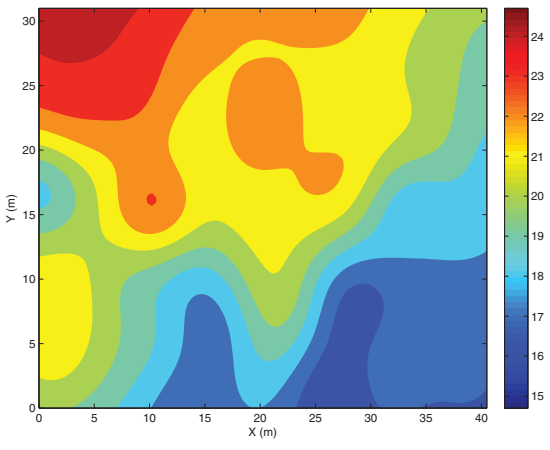

Fig. 3: Predicted temperature field at iteration 5

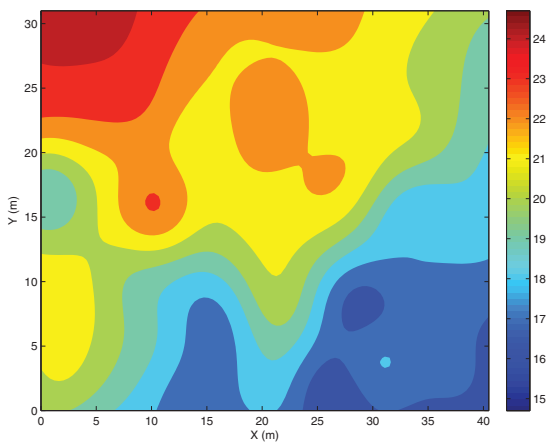

Fig. 4: Predicted temperature field at iteration 10

figures correspond to the starting locations.

In order to evaluate the effectiveness of the proposed approach, we compare the maximum predictive variance performance metric for the proposed method and random motion method. In random motion method, mobile robotic wireless sensors move along random trajectories. Note that after each iteration, sensors move to new locations and sample the environment. Following that, hyperparameters are learned and variances at all unobserved locations are continuously updated. In Fig. 8, we can see that the maximum predictive variance is degrading gradually as the number of iterations increases as sensors have better knowledge of the environment. In particular, it can be clearly seen that the proposed algorithm exhibits improved performance continuously as each successive motion of sensor nodes is driven by the information gain.

\section{CONCLUSION}

In this work we have considered the problem of prediction in spatial phenomena using observations obtained by a robotic mobile sensor network. We modeled the spatial field using Gaussian Processes and proposed a variance based density function for a loacational optimization problem. As the number of observations increases, the proposed computationally efficient algorithm allows the mobile sensors to find the most informative locations in collecting measurements minimizing the prediction error variance. The demonstrations of results on real-world datasets have shown that our proposed approach 


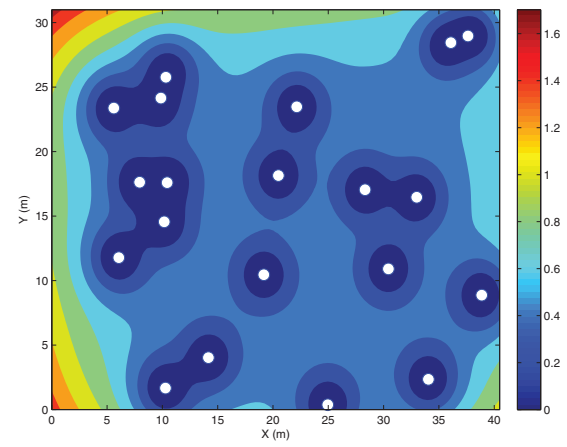

Fig. 5: Prediction error variances at iteration 1

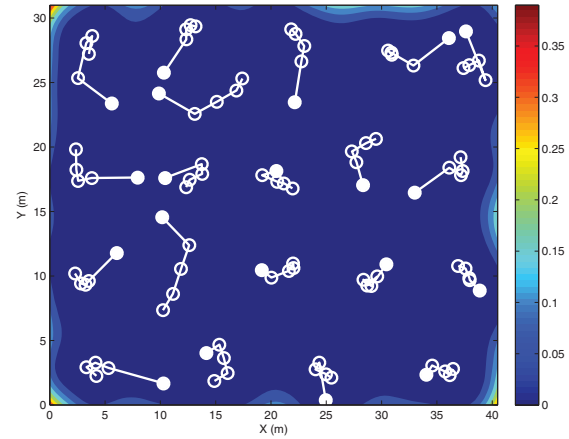

Fig. 6: Prediction error variances at iteration 5

outperforms the random motion method.

\section{REFERENCES}

[1] I. F. Akyildiz, W. Su, Y. Sankarasubramaniam, and E. Cayirci, Wireless sensor networks: a survey, Computer Networks, vol. 38, pp 393-422, 2002.

[2] L. F. Merino, J. R. Caballero, J. M. de Dios, and A. O. Ferruz, A Cooperative Perception System for Multiple UAVs: Application to Automatic Detection of Forest Fires, Journal of Field Robotics, 23(3), pp 165-184, 2006.

[3] C. E. Corrigan, G. C. Roberts, M. V. Ramana, D. Kim, and V. Ramanathan, Capturing Vertical Profiles of Aerosols and Black Carbon over the Indian Ocean Using Autonomous Unmanned Aerial Vehicles, Atmospheric Chemistry and Physics Discussions, 7(4), pp 11429-11463, 2007.

[4] N. E. Leonard, D. Paley, F. Lekien, R. Sepulchre, D. M. Fratantoni, and R. Davis, Collective Motion Sensor Networks and Ocean Sampling, IEEE Trans. on Robotics, 24(3), pp 710-724, 2007.

[5] N. A. Cressie, Statistics for spatial data, Wiley, 1991.

[6] A. Krause, A. Singh, and C. Guestrin, Near-Optimal Sensor Placement in Gaussian Processes: Theory, Efficient Algorithms and Empirical Studies, Journal of Machine Learning Research, 9, pp 235-284, 2008.

[7] C. Guestrin, A. Krause and A. P. Singh, "Near-optimal sensor placements in Gaussian Processes", in Proc. 22th International Conference on Machine Learning, Bonn, Germany, pp. 265-272, 2005.

[8] Y. Yang, and S. B. Rick, Sensor Placement in Gaussian Random Field Via Discrete Simulation Optimization, IEEE Signal Processing Letter, 15, pp 729-732, 2008.

[9] W. F. Caselton and J. V. Zidek, Optimal monitoring network designs, Statistics and Probability Letters, vol. 2, pp 223-227, 1984.

[10] C. Ko, J. Lee and M. Queyranne, An exact algorithm for maximum entropy sampling, Operations Research, vol. 43, pp 684-691, 1995.

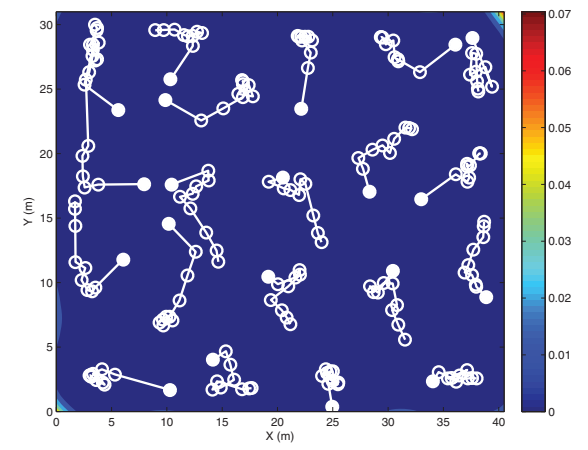

Fig. 7: Prediction error variances at iteration 10

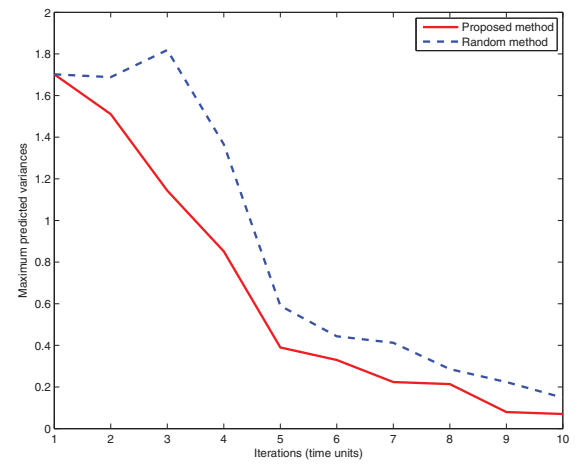

Fig. 8: Maximum prediction error variance

[11] L. Nguyen, S. Kodagoda, R. Ranasinghe, and G. Dissanayake, "Simulated annealing based approach for near-optimal sensor selection in Gaussian Processes" in Proc. IEEE International Conference on Control, Automation and Information Science (ICCAIS 2012), Ho Chi Minh city, Vietnam, November 2012.

[12] Z. Butler, and D. Rus, "Controlling mobile sensor for monitoring events with coverage constraints", in Proc. IEEE International Conference of Robotics and Automation, New Orleans, LA, pp. 1563-1573, 2004.

[13] J. Cortes, S. Martinez, T. Karatas, and F. Bullo, Coverage Control for Mobile Sensing Network, IEEE Transactions on Robotics and Automation, vol. 20, no. 2, pp. 243-254, 2004.

[14] Q. Du, V. Faber, and M. Gunzburger, Centroidal Voronoi Tessellation: Applications and Algorithms, SIAM Rev., vol. 41, no. 4, pp. 637-676, 1999.

[15] R. C. Hibbeler, Engineering Mechanics: Statics and Dynamics, 11th ed. Prentice Hall, ISBN 0132215098.

[16] F. Bullo, J. Cortes, and S. Martinez, Distributed Control of Robotic Networks, Applied Mathematics Series, Princeton University Press, 2009, ISBN 978-0-691-14195-4.

[17] C. E. Rasmussen and C. K. I. Williams, Gaussian Process for Machine Learning, The MIT Press, 2006.

[18] C. M. Bishop, Pattern Recognition and Machine Learning, Springer, New York, USA, 2006.

[19] F. Pukelsheim, Optimal Design of Experiments, volume 50 of Classics in Applied Mathematics. SIAM, Philadelphia, 2006.

[20] T. M. Cover, J. A. Thomas, Elements of Information Theory, Wiley and Sons, 2006.

[21] J. E. Goodman, J. O'Rourke, Handbook of Discrete and Computational Geometry, CRC Press, 2004.

[22] P. J. Ribeiro Jr. and P. J. Diggle, geoR: A package for geostatistical analysis, $R$ NEWS, vol. 1, 2001, pp 15-18. 